\title{
RIGIDITY OF INFINITE HEXAGONAL TRIANGULATION OF THE PLANE
}

\author{
TIANQI WU, XIANFENG GU, AND JIAN SUN
}

\begin{abstract}
In this paper, we consider the rigidity problem of the infinite hexagonal triangulation of the plane under the piecewise linear conformal changes introduced by Luo in 2004. Our result shows that if a geometric hexagonal triangulation of the plane is $\mathrm{PL}$ conformal to the regular hexagonal triangulation and all inner angles are in $[\delta, \pi / 2-\delta]$ for any constant $\delta>0$, then it is the regular hexagonal triangulation. This partially solves a conjecture of Luo. The proof uses the concept of quasi-harmonic functions to unfold the properties of the mesh.
\end{abstract}

\section{INTRODUCTION}

1.1. PL conformal. Given a smooth manifold $M$, two Riemannian metrics $g$ and $\tilde{g}$ are called conformally equivalent if

$$
\tilde{g}=e^{2 \lambda} g
$$

where $\lambda \in C^{\infty}(M)$ is called a conformal factor. In the discrete setting, Luo [5] introduced a notion of PL conformal equivalence of two piecewise linear polyhedral metrics in any dimension and developed a variational principle for PL conformality for triangulated surfaces with PL metrics [5]. Specifically, suppose the surface $\Sigma$ has a triangulation $T$, i.e., a $\mathrm{CW}$ complex whose faces are triangles which are glued edge-to-edge by isometrics. We denote such a triangulated surface by $(\Sigma, T)$, and its sets of vertices, edges, and triangles of $T$ by $V, E$ and $F$, respectively. Two triangulated surfaces $(\Sigma, T)$ and $(\tilde{\Sigma}, \tilde{T})$ are called combinatorially equivalent if there is a homeomorphism between $\Sigma$ and $\tilde{\Sigma}$ preserving the triangulation. For simplicity, we use the same notation to denote two combinatorially equivalent triangulated surfaces when the homeomorphism is not relevant or clear. Recall that a piecewise linear metric (or simply PL metric) on $(\Sigma, T)$ is a metric on $\Sigma$ so that its restriction to each triangle is isometric to a Euclidean triangle. It is uniquely determined by a function $\ell: E \rightarrow \mathbb{R}_{>0}$ which assigns a length to each edge so that the triangle inequalities hold for every triangle in $F$. With a PL metric, the triangulated surface $(\Sigma, T)$ is locally isometric to the Euclidean plane or half-plane if there is boundary except at the vertices where the metric may have cone-like singularities. In this paper, we will always assume a triangulated surface is equipped with some PL metric. We denote by $i j$ the edge with vertices $i$ and $j$, and by $i j k$ the triangle with vertices $i, j$ and $k$. If $f, g$ and $h$ are the functions over $V, E$, and $F$, respectively, for simplicity, we often write $f_{i}, g_{i j}$ and $h_{i j k}$ for $f(i), g(i j)$ and $h(i j k)$.

Received by the editors June 16, 2013 and, in revised form, September 13, 2013.

2010 Mathematics Subject Classification. Primary 52C25, 52C26. 


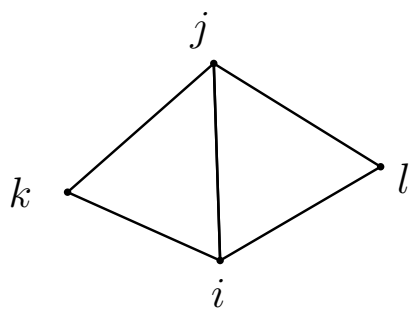

FigurE 1. The length cross ratio of the edge $i j$ is defined as $\frac{l_{i l} l_{j k}}{l_{i k} l_{j l}}$.

Definition 1.1 (Luo [5]). Two PL metrics $\ell$ and $\tilde{\ell}$ on combinatorially equivalent triangulated surfaces $(\Sigma, T)$ are PL conformal if

$$
\tilde{\ell}_{i j}=e^{w_{i}+w_{j}} \ell_{i j}
$$

for some function $w: V \rightarrow \mathbb{R}$ and for all edges $i j$.

The function $w$ plays an analogous role of conformal factor in this PL setting and thus is called a $P L$ conformal factor. We call such change of a PL metric on $(\Sigma, T)$ a $P L$ conformal change. This defines an equivalent relation on PL metrics on $(\Sigma, T)$. We call an equivalent class a $P L$ conformal class on $(\Sigma, T)$. Motivated by the smooth Yamabe problem, Luo [5] considered the existence and uniqueness of PL metrics with prescribed curvature in a PL conformal class. Namely, suppose $d$ is a PL metric on $(\Sigma, T)$ and $K: V \rightarrow \mathbf{R}$ is given. Is there a PL metric $d^{\prime}$ on $(\Sigma, T)$ which is PL conformal to $d$ so that the curvature of $d^{\prime}$ is $K$ (discrete Yamabe problem)? Is the metric $d^{\prime}$ unique up to scaling? There has been work done on solving both questions for finite triangulations of compact surfaces. The main issue that we address in this paper is a conjecture of Luo [4] about the uniqueness of the simplest infinite triangulation of the plane. Namely, suppose $d$ is a PL metric on the hexagonal triangulation of the plane so that (1) it is PL conformal to the regular hexagonal tiling, (2) the metric $d$ is complete and has zero curvature at each vertex. Is $d$ the regular hexagonal tiling? Our main result gives an affirmative answer to this question for those PL metrics so that all inner angles are in $(0, \pi / 2-\delta]$ for some $\delta>0$.

One can also look at the PL conformal transformation in terms of cross-ratio (see Bobenko, Pinkall and Springborn [1]). For an interior edge $i j$ incident to triangles $i j k$ and $i l j$ as in Figure 1, if the quadrilateral $i l j k$ is embedded in the complex plane $\hat{\mathbb{C}}=\mathbb{C} \cup\{\infty\}$ and we denote the vertex positions by $z_{i}, z_{j}, z_{k}, z_{l}$, any conformal map of $\hat{\mathbb{C}}$ preserves the cross ratio $\left(z_{i}, z_{j}, z_{l}, z_{k}\right):=\frac{z_{i}-z_{l}}{z_{i}-z_{k}} / \frac{z_{j}-z_{l}}{z_{j}-z_{k}}$. One can see that the absolute value of this cross ratio is $\frac{l_{i l} l_{j k}}{l_{i k} l_{j l}}$ (called length cross ratio by Bobenko, Pinkall and Springborn [1]) which is preserved by the PL conformal change since scale factors are cancelled.

Another motivation for Luo's conjecture comes from Thurston's conjecture on the rigidity of hexagonal circle packing in the plane. In his famous address 11 "The Finite Riemann Mapping Theorem", Thurston gave a different approach to PL conformal geometry for a triangulated surface using circle packings, which takes

\footnotetext{
${ }^{1}$ International Symposium in Celebration of the Proof of the Bieberbach Conjecture. Purdue University, March 1985.
} 


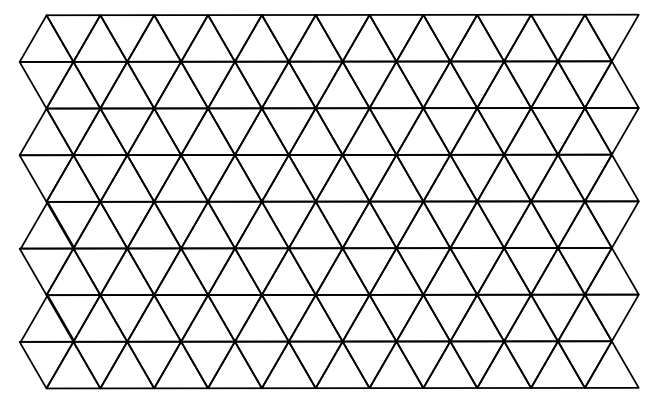

FIGURE 2. Regular triangulation of the plane.

the view of the conformal map preserving infinitesimal circles. In Definition 1.1. if we let $\widetilde{l_{i j}}=\left(e^{w_{i}}+e^{w_{j}}\right)$, we obtain a circle packing metric $\widetilde{l_{i j}}$ in the sense of Thurston. Thurston conjectured, among other things, that the only complete flat circle packing metric on the hexagonal triangulation of the plane is the regular hexagonal packing. This was proved by Rodin and Sullivan [6]. The main problem that we study is the counterpart of Thurston's conjecture in the new PL conformal setting.

In the circle packing setting, it is natural to assign each vertex $i$ a circle with the radius $e^{w_{i}}$, and then the circles centered at two neighboring vertices are tangential to each other. This PL conformal transformation map circles to circles, or in an equivalent way. The identity $l_{i l}+l_{j k}=l_{i k}+l_{j l}$ always holds for any edge $i j$. This identity also holds for conformal transformation on $\hat{\mathbb{C}}$. One disadvantage of this approach is that in general the meshes we get in practice do not satisfy $l_{i l}+l_{j k}=l_{i k}+l_{j l}$, and usually we have to give up its edge-length information by assuming $l \equiv$ const to do the PL conformal transformation. However, in the new PL conformality the initial metric information can be reserved and the transformation can be done directly with the original mesh.

Let $\alpha_{i}$ be the cone angle at the vertex $i$ which is the sum of all inner angles having the vertex $i$. The curvature is a function over the vertices: $K: V \rightarrow \mathbb{R}$ defined by $K_{i}=2 \pi-\alpha_{i}$ if the vertex $i$ is in the interior and $K_{i}=\pi-\alpha_{i}$ if the vertex $i$ is on the boundary. It is obvious that the curvature is uniquely determined by the PL metric. Its converse is the type of rigidity problem (uniqueness question) we consider here: is the PL metric in a given PL conformal class uniquely determined up to a scaling (i.e., an Euclidean similar transformation) by the curvature function? In this paper, we will focus on the triangulated planes with the curvature function $K \equiv 0$, or the triangulated flat planes for short. Notice that a triangulated flat plane may not be isometric to the Euclidean plane.

1.2. Problem and main results. Below is the main problem which was first studied in [5].

Given two $P L$ metrics $\ell$ and $\tilde{\ell}$ on combinatorially equivalent triangulated surfaces $(\Sigma, T)$ so that both metrics are isometric to the complex plane $\mathbb{C}$, if $\ell$ and $\tilde{\ell}$ are $P L$ conformally equivalent, do they differ by a scaling?

In this paper, we restrict ourselves to the hexagonal triangulation of the plane where every vertex is of degree 6 . The regular hexagonal triangulation is the tiling of the plane by regular equilateral triangles as shown in Figure 2 i.e., we assume a 

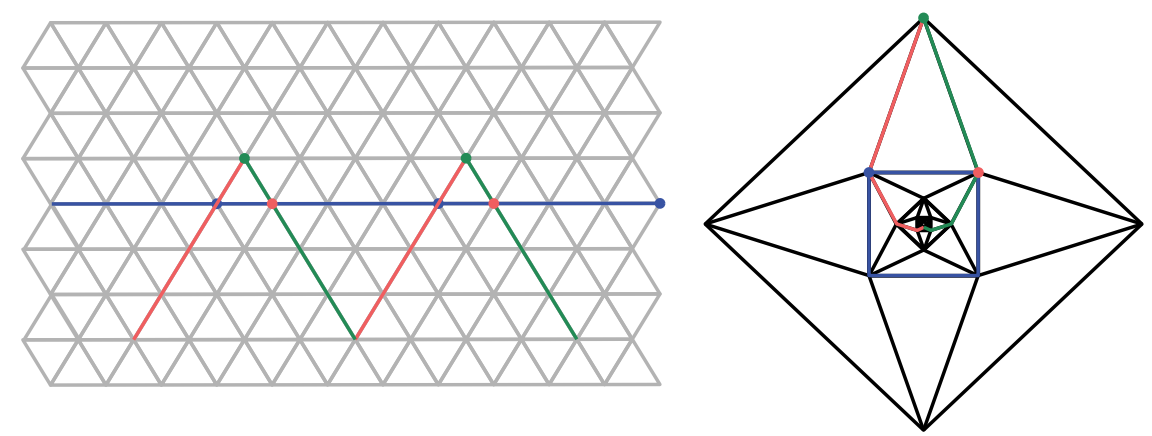

Figure 3. A hexagonal triangulation of the flat plane. (Color available online.)

regularly triangulated plane is equipped with a PL metric where all the edges have the same length. It is obvious that a regularly triangulated plane is isometric to the complex plane. The main result of this paper is the following rigidity theorem.

Theorem 1. If a hexagonally triangulated plane with a piecewise flat metric $\ell$ satisfies the following conditions:

(1) it is PL conformal to the regularly triangulated plane,

(2) it is isometric to the complex plane $\mathbb{C}$, and

(3) $\sup \{$ all inner angles of the triangulation $\}<\pi / 2$,

then it has to be regular, i.e., $\ell$ is constant, or equivalently, the conformal factor $w$ is constant.

Although our proof of the theorem relies on condition (3), we believe that it is not a necessary hypothesis and conjecture the theorem still holds even without it.

Condition (2) is stronger than the flat condition that curvature $K=0$. Indeed, there are incomplete flat PL metrics on the plane. Condition (2) is equivalent to the complete flatness. We call the conformal factor $w$ linear if it is the restriction of a linear function on $\mathbb{C}$ to the vertices in a regular triangulation. One can show that a hexagonally triangulated plane is flat if it is conformal to a regular one with a linear PL conformal factor $w$ (see Lemma 2.5). This gives a two-parameter family of the flat planes of hexagonal triangulation. Other than the regular ones, each of them must have an overlap of positive area if it is immersed into the complex plane.

Figure 3 shows such an example with a linear PL conformal factor $w$. The left picture shows the regular hexagonal triangulation of $\mathbb{C}$. The mesh in the right figure contains a nested infinite sequence of squares so that the $i$ th square is transformed to the $(i+1)$ th square by a rotation of $\pi / 2$ and a scaling whose ratio is independent of $i$. One can choose this ratio so that the length cross ratio of each edge in the mesh is 1 . This mesh becomes a hexagonal triangulation of $\mathbb{C} \backslash\{0\}$. Extend this mesh to the universal covering space of $\mathbb{C} \backslash\{0\}$, and obtain a flat plane with the hexagonal triangulation where the length cross ratio of each edge is 1 . Thus this extension of the mesh in the right figure is PL conformal to the regularly triangulated plane. In Figure 3, the colored edges on the left correspond to the edges of the same color on the right. One can verify that the conformal factor $w$ is linear based on the similarity relation of the triangles in the mesh. It is shown in Lemma 2.6 that this extension 
of the mesh in the right figure cannot be immersed to $\mathbb{C}$ without overlapping. We conjecture that this two-parameter family characterizes all hexagonal triangulation of the flat plane conformal to the regular one.

Conjecture 1. A hexagonally triangulated flat plane is conformal to the regular one if and only if the PL conformal factor $w$ is linear.

The rigidity of the PL conformal transformation for a compact finitely triangulated surface was initially investigated by Luo [5] where he proved that the metric within a PL conformal class is locally uniquely determined by the curvature by establishing a variational principle whose action functional is a locally convex function. In 2010, Bobenko, Pinkall and Springborn [1 found, among other things, an explicit formula for the action functional and showed that it extends to a globally convex one. Using this, they proved a global rigidity for finite triangulated surfaces. However, the variational method cannot be extended to show the rigidity problem for infinite triangulated surfaces since the action functional may become infinite.

A similar rigidity result has been proved in the infinite circle packing case. Rodin and Sullivan [6] and He 2] proved the rigidity of hexagonal circle packings of the complex plane and their methods can be extended to prove the rigidity of packings with bounded valence. Schramm [7] used a topological property to extend the rigidity for the packings of arbitrary (locally finite) planar triangulations. He [3] showed that a variation along two conformal packings is a harmonic function on a recurrent network and hence is constant. In this way, he proved the rigidity of packings even with overlaps.

\section{Outline of the PRoOF}

In this section we outline the proof of Theorem 1 . With a properly chosen coordinate system, one can index by a couple of integers $(m, n)$ the vertices in the regular triangulation with the edge length equal to 1 :

$$
V_{0}=\left\{m+n \omega \mid m, n \in \mathbb{Z}, \omega=-\frac{1}{2}+\frac{\sqrt{3}}{2} i\right\} .
$$

Since any hexagonal triangulation of the plane $(\Sigma, T)$ has the same combinatorial structure as this regular one, we also index the vertices $V$ of any hexagonal triangulation in the same manner. We write $i \sim j$ if $i, j \in V$ are two endpoints of an edge in $E$.

The metric of a hexagonally trangulated plane $(\Sigma, T)$ conformal to a regular one is uniquely determined by the PL conformal factor $w$ up to a similarity. Condition (2) in Theorem 1 that $(\Sigma, T)$ is isometric to $\mathbb{C}$ implies that the curvature induced by the metric is everywhere 0 , which in return imposes certain restrictions on the function $w$. We introduce a conception called quasi-harmonic and show the function $w$ is quasi-harmonic.

Definition 2.1. We say a function $f$ on $V$ is quasi-harmonic if there exists $m>0$ depending on $f$ such that, for any $i \in V$, with its six neighbors $i_{1}, i_{2}, \cdots, i_{6}$, there exist $m_{1}^{i}, m_{2}^{i}, \cdots, m_{6}^{i} \geq m$, satisfying

$$
\sum_{j=1}^{6} m_{j}^{i}=1 \quad \text { and } \quad f(i)=\sum_{j=1}^{6} m_{j}^{i} f\left(i_{j}\right) .
$$

We call such $m$ a harmonic factor of $f$. 
We also define the discrete difference of the PL conformal factor $w$ as follows.

Definition 2.2. For any $c \in V$, we define the difference of $w$ with vector $c$ as $\Delta_{c} w(i)=w(i+c)-w(i)$.

With this definition of quasi-harmonic and the difference operator, we have the following lemma.

Lemma 2.1. If a hexagonally triangulated flat plane $(\Sigma, T)$ is $P L$ conformal to a regular one and

$$
\sup \{\text { all inner angles of the triangulation }\}=\theta<\pi / 2 \text {, }
$$

then for any constant $c \in V$, the function $\Delta_{c} w$ is quasi-harmonic and its harmonic factor $m(\theta)$ depends only on $\theta$.

By definition, if $m$ is a harmonic factor of a quasi-harmonic function $f$, any $\widetilde{m} \in$ $(0, m)$ is also a harmonic factor. It should be noted that quasi-harmonicity is weaker than harmonicity related to the graph Laplace operator. In quasi-harmonicity the weight $m_{i}^{j}$ is directed, i.e., $m_{i}^{j}$ is not necessarily equal to $m_{j}^{i}$. This means the random walk on the 1-skeleton of $T$ defined by the weights $m_{i}^{j}$ may not be reversible. Nevertheless, a quasi-harmonic function satisfies the maximal principle. Note that it is only Lemma 2.1 whose proof in this paper requires condition (3) that all inner angles of the hexagonal triangulation are strictly acute.

It is well known that a bounded harmonic function on a recurrent network must be constant ([3, Lemma 5.5]). We show that a quasi-harmonic function on a network has a similar property of almost constant over an arbitrarily large region. We assume a graph distance between any two vertices $i, j$ in $V$, i.e.,

$$
d(i, j)=\inf \{t \in N \mid \exists \text { a path with } t \text { edges in } E \text { connecting } i \text { and } j\} .
$$

We denote $B(i, R)=\{j \in V \mid d(i, j) \leq R\}$. Note that in the definition we use $\leq$ instead of $<$ and it's a little different from the continuous case.

Lemma 2.2. Given a quasi-harmonic function $f$ on $V$ with harmonic factor $m$, if there exist $M \in \mathbb{R}, R>0, \epsilon>0$ and $i \in V$ so that

$$
f(i) \geq M-\epsilon m^{R} \quad \text { and }\left.\quad f\right|_{B(i, R)} \leq M,
$$

we have $\left.f\right|_{B(i, R)} \geq M-\epsilon$.

In the case where the given quasi-harmonic function $f$ is bounded, we can choose $M$ as its least upper bound and for any $R, \epsilon>0$ choose vertex $i$ satisfying $f(i) \geq$ $M-\epsilon m^{R}$, and then by Lemma 2.2 we have $\left.f\right|_{B(i, R)} \geq M-\epsilon$. This shows there is an arbitrarily large region (specified by $R$ ) where a bounded quasi-harmonic function is a constant up to an arbitrarily small perturbation (specified by $\epsilon$ ). Based on Lemma 2.2. we can prove a stronger result which says there is an arbitrarily large region where any two bounded quasi-harmonic functions are simultaneously constant up to an arbitrarily small perturbation.

Lemma 2.3. Given two bounded quasi-harmonic functions $f_{1}, f_{2}$ on $V$, assume the least upper bound of $f_{1}$ is $M$. Then for any $R>0, \epsilon>0$, there exists $N \in \mathbb{R}$ and $i \in V$ such that

$$
\begin{gathered}
M-\epsilon \leq\left. f_{1}\right|_{B(i, R)} \leq M \\
N-\epsilon \leq\left. f_{2}\right|_{B(i, R)} \leq N
\end{gathered}
$$


Given a PL conformal factor $w$ on the vertices $V$, we focus on two functions $\Delta_{1} w$ and $\Delta_{\omega} w$. The following lemma claims both functions are indeed bounded.

Lemma 2.4. If a hexagonally triangulated flat plane $(\Sigma, T)$ is $P L$ conformal to a regular one, then $\sup _{i \sim j}|w(j)-w(i)|<\infty$.

This, together with Lemma 2.1 shows both $\Delta_{1} w$ and $\Delta_{\omega} w$ are bounded quasiharmonic under the hypotheses of Theorem 11. Thus there is a large region where both functions are close constants. To see the consequence of this fact, we first investigate that of both $\Delta_{1} w$ and $\Delta_{\omega} w$ being exactly constant, or equivalently, $w$ being linear. The following lemma claims that the linearity of $w$ implies the flatness of the plane.

Lemma 2.5. If it is conformal to a regular one with a linear PL conformal factor, then a hexagonally triangulated plane $(\Sigma, T)$ has to be flat.

As we mentioned in the introduction section, this shows that there exists a twoparameter family of the flat plane conformal to a regular one where $w$ is induced by any linear function $a x+b y$ on the complex plane with $a, b \in \mathbb{R}$. However, we show that only if the function $w$ is constant can the flat plane isometrically be embedded into the complex plane.

Lemma 2.6. Assume $(\Sigma, T)$ is a hexagonally triangulated plane and conformal to a regular one, with $P L$ conformal factor $w$, if there exist $M>0, N \in \mathbb{R}$, so that

$$
\Delta_{1} w \equiv M \quad \text { and } \quad \Delta_{\omega} w \equiv N .
$$

Then there exists $R(M, N)>0$ depending on $M, N$ such that for any $i \in V$, $B(i, R) \subseteq V$ cannot be isometrically immersed into $\mathbb{C}$ without an overlap of positive area.

Based on the above result, we are able to show the following lemma if the PL conformal factor $w$ is nearly linear over a large region.

Lemma 2.7. Assume $(\Sigma, T)$ is a hexagonally triangulated flat plane and conformal to a regular one, with $P L$ conformal factor $w$, and all inner angles of the triangulation are in a compact set $S \subseteq(0, \pi)$. For any $M>0$, there exist $\epsilon>0, R>0$ only depending on $M, S$ such that for any $N \in \mathbb{R}, i \in V$ with

$$
M-\epsilon \leq\left.\Delta_{1} w\right|_{B(i, R)} \leq M \quad \text { and } \quad N-\epsilon \leq\left.\Delta_{\omega} w\right|_{B(i, R)} \leq N,
$$

$B(i, R) \subseteq V$ cannot be isometrically embedded into $\mathbb{C}$ without an overlap of positive area.

With the above lemmas, we are ready to prove Theorem 1 .

Proof of Theorem 1. By Lemma 2.1 and Lemma 2.4. $\Delta_{1} w$ and $\Delta_{\omega} w$ are both bounded quasi-harmonic. If $w$ is not constant, we may assume $M=\sup \left\{\Delta_{1} w\right\}>0$. By condition (3), we have that all inner angles are in $[\pi-2 \theta, \theta] \subseteq(0, \pi)$. Choose $R>0, \epsilon>0$ depending on $M$ and $\theta$ according to Lemma 2.7. Once $R$ and $\epsilon$ are chosen, one can choose $N$ and $i$ according to Lemma 2.3 such that the PL conformal factor $w$ satisfies

$$
M-\epsilon \leq\left.\Delta_{1} w\right|_{B(i, R)} \leq M \quad \text { and } \quad N-\epsilon \leq\left.\Delta_{\omega} w\right|_{B(i, R)} \leq N .
$$

Now by Lemma 2.7 this implies the part of the plane covering $B(i, R) \subseteq V$ cannot be isometrically embedded into $\mathbb{C}$ without an overlap of positive area. This reaches 


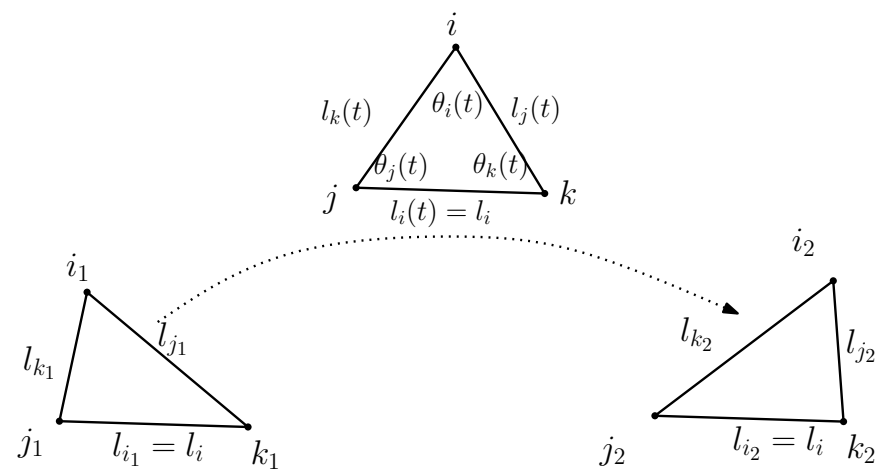

FiguRE 4. An acute triangle can be deformed to the target acute triangle in a monotonic way.

a contradiction to $(\Sigma, T)$ being isometric to $\mathbb{C}$. Therefore the PL conformal factor $w$ must be constant.

\section{Properties of $w$ (Lemmas 2.1 and 2.4)}

3.1. Proof of Lemma 2.1. We first show the following two lemmas. Lemma 3.1 shows that when an edge is fixed, one acute triangle can be deformed to a target acute triangle in a monotonic way (see Figure 4). Lemma 3.2 describes a relation between the angles and the edge lengths of two triangles.

Lemma 3.1. $\left\{i_{1}, j_{1}, k_{1}\right\},\left\{i_{2}, j_{2}, k_{2}\right\}$ are two triangles with each inner angle $<$ $\pi / 2$ and $l_{i_{1}}=l_{i_{2}}$. There exists a flow deforming $\left\{i_{1}, j_{1}, k_{1}\right\}$ to $\left\{i_{2}, j_{2}, k_{2}\right\}$ with corresponding edge length $l_{i}(t), l_{j}(t), l_{k}(t)$ satisfying

(1) $l_{i}(t)=l_{i_{1}}=l_{i_{2}}$;

(2) $l_{j}(0)=l_{j_{1}}, l_{j}(1)=l_{j_{2}}$;

(3) $l_{k}(0)=l_{k_{1}}, l_{k}(1)=l_{k_{2}}$;

(4) $l_{j}(t), l_{k}(t), \theta_{i}(t), \theta_{j}(t), \theta_{k}(t)$ are monotonic, continuous and piecewise differentiable where $\theta_{i}(t), \theta_{j}(t), \theta_{k}(t)$ are the three inner angles.

Lemma 3.2. For any angle $\theta<\pi / 2$ there exist $0<m(\theta)<M(\theta)$ such that for any two triangles $\left\{i_{1}, j_{1}, k_{1}\right\},\left\{i_{2}, j_{2}, k_{2}\right\}$ with all the inner angles no larger than $\theta$ and edge length $l_{i_{2}}=l_{i_{1}}, l_{j_{2}}=l_{j_{1}} e^{\tilde{u}_{j}}, l_{k_{2}}=l_{k_{1}} e^{\tilde{u}_{k}}$, there exist $a, b$ satisfying $m(\theta) \leq a, b \leq M(\theta)$ and $\theta_{i_{2}}-\theta_{i_{1}}=-a \tilde{u}_{j}-b \tilde{u}_{k}$.

We will prove Lemma 2.1 in the following three steps: (1) show Lemma 2.1 by assuming Lemma 3.2 holds; (2) show Lemma 3.2 by assuming Lemma 3.1 holds; (3) show Lemma 3.1 .

3.1.1. Proof of Lemma 2.1 provided Lemma 3.2 holds. Assume $i_{1}, i_{2}, \ldots, i_{6}$ are six neighbors of $i$ arranged counterclockwise, and then $i_{1}+c, i_{2}+c, \ldots, i_{6}+c$ are six neighbors of $i+c$ arranged counterclockwise. We denote the angle of $i$ in triangle $\{i, j, k\}$ as $\theta_{i}^{j k}$. For simplicity, when $i$ is fixed, we denote $\theta_{i}^{i_{j} i_{j+1}}=\theta_{i}^{j}$, $\theta_{i+c}^{\left(i_{j}+c\right)\left(i_{j+1}+c\right)}=\theta_{i+c}^{j}$ (assume $\left.i_{7}=i_{1}\right)$ (see Figure [5). 

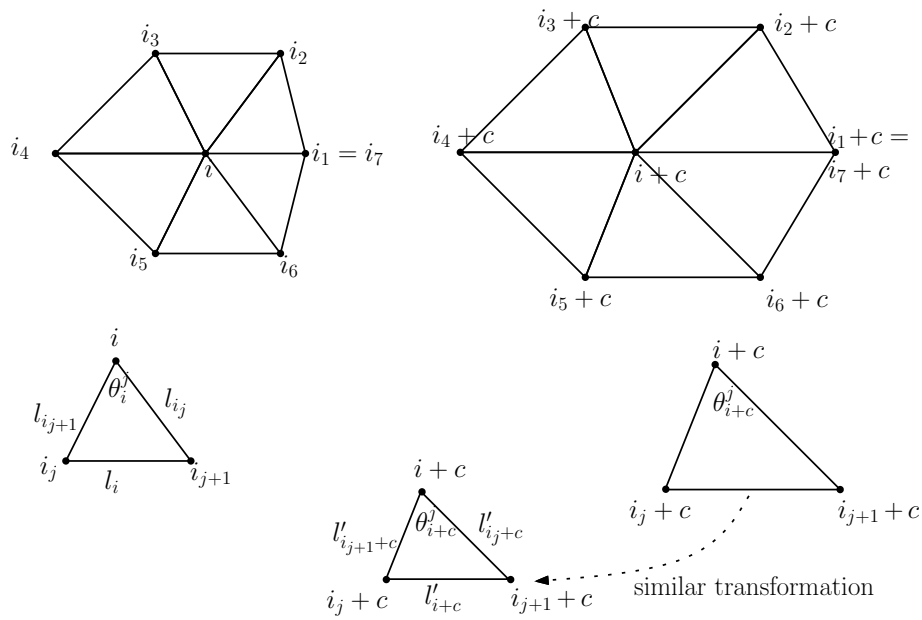

FiguRE 5. An acute triangle can be deformed to the target acute triangle in a monotonic way.

We consider two triangles $\triangle i i_{j} i_{j+1}, \triangle(i+c)\left(i_{j}+c\right)\left(i_{j+1}+c\right)$ (see Figure 5). Perform a similar transformation on the latter so that $l_{i+c}^{\prime}=l_{i}$ and we obtain

$$
\begin{aligned}
& l_{i_{j}+c}^{\prime}=l_{i_{j}} e^{\Delta_{c} w(i)-\Delta_{c} w\left(i_{j+1}\right)} \text { and } \\
& l_{i_{j+1}+c}^{\prime}=l_{i_{j+1}} e^{\Delta_{c} w(i)-\Delta_{c} w\left(i_{j}\right)} .
\end{aligned}
$$

By applying Lemma 3.2 to $\triangle i i_{j} i_{j+1}$ and the similarly transformed version of $\triangle(i+c)\left(i_{j}+c\right)\left(i_{j+1}+c\right)$, we have

$$
\theta_{i+c}^{j}-\theta_{i}^{j}=-a_{j}\left(\Delta_{c} w(i)-\Delta_{c} w\left(i_{j}\right)\right)-b_{j}\left(\Delta_{c} w(i)-\Delta_{c} w\left(i_{j+1}\right)\right)
$$

where $m(\theta) \leq a_{j}, b_{j} \leq M(\theta), m(\theta), M(\theta)$ are determined by $\theta$ only. Sum the equality above over all $j=1,2, \ldots, 6$, and we obtain

$$
\begin{aligned}
0 & =\sum_{j=1}^{6}\left(\theta_{i+c}^{j}-\theta_{i}^{j}\right) \quad(\text { for } K(i)=K(i+c)=0) \\
& =-\sum_{j=1}^{6}\left(a_{j}\left(\Delta_{c} w(i)-\Delta_{c} w\left(i_{j}\right)\right)+b_{j}\left(\Delta_{c} w(i)-\Delta_{c} w\left(i_{j+1}\right)\right)\right)
\end{aligned}
$$

which leads to

$$
\Delta_{c} w(i)=\sum_{j=1}^{6} \frac{a_{j}+b_{j-1}}{\sum_{j=1}^{6}\left(a_{j}+b_{j}\right)} \Delta_{c} w\left(i_{j}\right) \quad\left(b_{0}=b_{6}\right)
$$

where

$$
\frac{a_{j}+b_{j-1}}{\sum_{j=1}^{6}\left(a_{j}+b_{j}\right)}>\frac{2 m(\theta)}{12 M(\theta)}=\frac{m(\theta)}{6 M(\theta)} \quad \text { and } \quad \sum_{j=1}^{6} \frac{a_{j}+b_{j-1}}{\sum_{j=1}^{6}\left(a_{j}+b_{j}\right)}=1 .
$$

Therefore $\Delta_{c} w$ is quasi-harmonic and $\frac{m(\theta)}{6 M(\theta)}$ is its harmonic factor. 
3.1.2. Proof of Lemma 3.2 provided Lemma 3.1 holds. From Lemma 3.1 there exists a monotonic flow deforming $\left\{i_{1}, j_{1}, k_{1}\right\}$ to $\left\{i_{2}, j_{2}, k_{2}\right\}$. Assume $u_{j}(t)$ and $u_{k}(t)$ are functions on $[0,1]$ satisfying

$$
l_{j}(t)=l_{j_{1}} e^{u_{j}(t)} \quad \text { and } \quad l_{k}(t)=l_{k_{1}} e^{u_{k}(t)} .
$$

It is easy to verify that $u_{j}(0)=u_{k}(0)=0, u_{j}(1)=\tilde{u}_{j}, u_{k}(1)=\tilde{u}_{k}$. It can be calculated that

$$
\frac{\partial \theta_{i}}{\partial u_{j}}=-\cot \theta_{k} \quad \text { and } \quad \frac{\partial \theta_{i}}{\partial u_{k}}=-\cot \theta_{j}
$$

By the properties of the deforming flow stated in Lemma 3.1. we have that $u_{j}(t), u_{k}(t)$ are monotonic, continuous and piecewise differentiable, and all the inner angles remain in the interval $[\pi-2 \theta, \theta]$ during the flow. Thus we can apply the integral mean value theorem and obtain

$$
\begin{aligned}
\theta_{i_{2}}-\theta_{i_{1}} & =\theta_{i}(1)-\theta_{i}(0) \\
& =\int_{0}^{1} \frac{d \theta_{i}}{d t} d t \\
& =\int_{0}^{1}\left(\frac{\partial \theta_{i}}{\partial u_{j}} \frac{d u_{j}}{d t}+\frac{\partial \theta_{i}}{\partial u_{k}} \frac{d u_{k}}{d t}\right) d t \\
& =\int_{0}^{1}\left(-\cot \theta_{k}\right) \frac{d u_{j}}{d t} d t+\int_{0}^{1}\left(-\cot \theta_{j}\right) \frac{d u_{k}}{d t} d t \\
& =-\cot \theta_{k}\left(x_{k}\right) \int_{0}^{1} \frac{d u_{j}}{d t} d t-\cot \theta_{j}\left(x_{j}\right) \int_{0}^{1} \frac{d u_{k}}{d t} d t \quad\left(0 \leq x_{j}, x_{k} \leq 1\right) \\
& =-\cot \theta_{k}\left(x_{k}\right) u_{j}(1)-\cot \theta_{j}\left(x_{j}\right) u_{k}(1) .
\end{aligned}
$$

Let $m(\theta)=\cot \theta, M(\theta)=\cot (\pi-2 \theta)$. As $\theta_{j}(t)$ and $\theta_{k}(t)$ remain in the interval $[\pi-2 \theta, \theta]$ for all $t \in[0,1]$, we have $m(\theta) \leq \cot \theta_{k}\left(x_{k}\right), \theta_{j}\left(x_{j}\right) \leq M(\theta)$. This proves the lemma.

We remark that here we need the acute triangle assumption to ensure that $\cot \theta_{k}(t), \cot \theta_{k}(t)>0$ are positive and bounded.

3.1.3. Proof of Lemma 3.1. As $l_{i}$ is fixed in this deforming flow, $\theta_{i}, \theta_{j}$ and $\theta_{k}$ are the functions of $l_{j}$ and $l_{k}$. Conversely, $l_{j}$ and $l_{k}$ are the functions $\theta_{j}$ and $\theta_{k}$. Note that the triangles are assumed to be acute and therefore $\cot \theta_{i}, \cot \theta_{j}$ and $\cot \theta_{k}$ are all positive. It can be calculated that

$$
\begin{aligned}
& \frac{\partial \theta_{j}}{\partial l_{j}}=\frac{\cot \theta_{i}+\cot \theta_{k}}{l_{j}}>0, \quad \frac{\partial \theta_{k}}{\partial l_{j}}=-\frac{\cot \theta_{i}}{l_{j}}<0, \\
& \frac{\partial \theta_{k}}{\partial l_{k}}=\frac{\cot \theta_{i}+\cot \theta_{j}}{l_{k}}>0, \quad \frac{\partial \theta_{i}}{\partial l_{k}}=-\frac{\cot \theta_{j}}{l_{i}}<0, \\
& \frac{\partial \theta_{i}}{\partial l_{j}}=-\frac{\cot \theta_{k}}{l_{i}}<0 \quad \text { and } \quad \frac{\partial \theta_{i}}{\partial l_{k}}=-\frac{\cot \theta_{j}}{l_{i}}<0 .
\end{aligned}
$$


Similarly, we have

$$
\begin{array}{clrl}
\frac{\partial l_{j}}{\partial \theta_{j}}=l_{j}\left(\cot \theta_{j}+\cot \theta_{i}\right)>0, & \frac{\partial l_{j}}{\partial \theta_{k}}=l_{j} \cot \theta_{i}>0 \\
\frac{\partial l_{k}}{\partial \theta_{k}}=l_{k}\left(\cot \theta_{k}+\cot \theta_{i}\right)>0 & \text { and } & \frac{\partial l_{k}}{\partial \theta_{j}}=l_{k} \cot \theta_{i}>0 .
\end{array}
$$

We prove the lemma by specifying the deforming flow for different cases as follows:

(1) If $\theta_{j}(0) \leq \theta_{j}(1)$ and $\theta_{k}(0) \leq \theta_{k}(1)$, we can choose the flow such that $\theta_{j}(t)$ and $\theta_{k}(t)$ are both linear. This means $\theta_{i}(t)$ is linear too. Thus $\theta_{j}^{\prime}(t) \geq 0$, $\theta_{k}^{\prime}(t) \geq 0$ and $\theta_{i}(t), \theta_{j}(t), \theta_{k}(t)$ are all acute for any $t$. Therefore $\frac{d l_{j}}{d t}=$ $\frac{\partial l_{j}}{\partial \theta_{j}} \theta_{j}^{\prime}+\frac{\partial l_{k}}{\partial \theta_{k}} \theta_{k}^{\prime} \geq 0$ and $\frac{d l_{k}}{d t}=\frac{\partial l_{k}}{\partial \theta_{j}} \theta_{j}^{\prime}+\frac{\partial l_{k}}{\partial \theta_{k}} \theta_{k}^{\prime} \geq 0$.

(2) If $\theta_{j}(0) \geq \theta_{j}(1)$ and $\theta_{k}(0) \geq \theta_{k}(1)$, this case is similar to case (1).

(3) If $l_{j}(1)=l_{j}(0)$, we choose $l_{j}(t)=l_{j}(0)$ and $l_{k}(t)$ is linear. Now we show that $\theta_{i}(t), \theta_{j}(t)$ and $\theta_{k}(t)$ are monotonous. It suffices to show they are monotonic in variable $l_{k}$. $\frac{\partial \theta_{k}}{\partial l_{k}}=\left(\cot \theta_{j}+\cot \theta_{i}\right) / l_{k}>0$ as $\theta_{i}+\theta_{j}<\pi$. Note that we have not shown the acuteness of $\theta_{j}$ and $\theta_{i}$. Without loss of generality we may assume that $l_{i} \geq l_{j}$, and thus $\theta_{j}(t)<\pi / 2$. We have $\frac{\partial \theta_{i}}{\partial l_{k}}=-\cot \theta_{j} / l_{k}<0$ and thus $\theta_{i}$ is monotonic. This forces that $\theta_{i}(t)$ lies between $\theta_{i}(0)$ and $\theta_{i}(1)$ and thus $\cot \theta_{i}(t)>0$ for any $0<t<1$. Therefore $\frac{\partial \theta_{j}}{\partial l_{k}}=-\cot \theta_{i} / l_{k}<0$ and $\theta_{j}$ is monotonic.

(4) If $l_{k}(0)=l_{j}(1)$, this case is similar to case (3).

(5) If $\theta_{j}(0) \leq \theta_{j}(1), \theta_{k}(0) \geq \theta_{k}(1), l_{j}(0) \leq l_{j}(1)$ and $l_{k}(0) \geq l_{k}(1)$, we keep angle $\theta_{i}$ fixed, and let $\theta_{j}$ increase and $\theta_{k}$ decrease at the same rate. This can be done by moving the vertex $i$ along the circumscribing circle of $\triangle i_{1} j_{1} k_{1}$. Based on the sine law, $l_{j}$ increases and $l_{k}$ decreases. All the quantities are moving close to their counterparts in $\triangle i_{2} j_{2} k_{2}$. Stop once one of the following happens: (i) $l_{j}(t)$ reaches $l_{j}(1)$, or (ii) $l_{k}(t)$ reaches $l_{k}(1)$, or (iii) $\theta_{j}(t)$ reaches $\theta_{j}(1)$, or (iv) $\theta_{k}(t)$ reaches $\theta_{k}(1)$. Note that one of the above four cases must happen at some point $t$, which is a previously discussed case (1 or 2 or 3 or 4 ).

(6) If $\theta_{j}(0) \geq \theta_{j}(1), \theta_{k}(0) \leq \theta_{k}(1), l_{j}(0) \geq l_{j}(1)$ and $l_{k}(0) \leq l_{k}(1)$, this case is similar to case $(5)$.

Here we remark that the case where $\theta_{i}(0)=\theta_{i}(1)$ must fall into either case $(5)$ or case $(6)$.

(7) If $\theta_{j}(0)>\theta_{j}(1), \theta_{k}(0)<\theta_{k}(1), l_{j}(0)<l_{j}(1)$ and $l_{k}(0)<l_{k}(1)$, we fix $l_{j}$ and increase $\theta_{k}$. From the cosine law we have that $l_{k}$ increases at the same time. By the same reasoning as in case (3), both $\theta_{i}$ and $\theta_{j}$ decrease at the same time. We stop deforming once one of the following happens: (i) $\theta_{i}$ reaches $\theta_{i}(1)$, or (ii) $\theta_{j}$ reaches $\theta_{j}(1)$, or (iii) $\theta_{k}$ reaches $\theta_{k}(1)$, or (iv) $l_{k}$ reaches $l_{k}(1)$. Again, during the deformation, all the quantities are moving close to their counterparts in $\triangle i_{2} j_{2} k_{2}$. Thus one of the above four cases must happen at some point $t$, which is a previously discussed case.

(8) There are three cases left: (i) $\theta_{j}(0)<\theta_{j}(1), \theta_{k}(0)>\theta_{k}(1), l_{j}(0)>l_{j}(1)$ and $l_{k}(0)>l_{k}(1)$, or (ii) $\theta_{j}(0)<\theta_{j}(1), \theta_{k}(0)>\theta_{k}(1), l_{j}(0)<l_{j}(1)$ and $l_{k}(0)<l_{k}(1)$, or (iii) $\theta_{j}(0)>\theta_{j}(1), \theta_{k}(0)<\theta_{k}(1), l_{j}(0)>l_{j}(1)$ and $l_{k}(0)>l_{k}(1)$. They are similar to case $(7)$. 


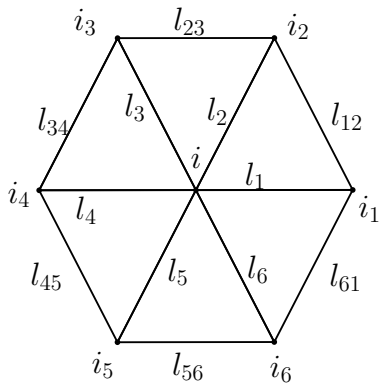

FiguRE 6. Illustration for the proof of Lemma 2.4.

3.2. Proof of Lemma 2.4. We prove $\Delta_{1} w(i)=w(i+1)-w(i)$ has a lower bound and the other inequalities can be shown in a similar way. Let $i_{1}, i_{2}, \ldots, i_{6}$ be six neighbors of $i$ and $i_{1}=i+1$ (see Figure 6). Let $e^{w\left(i_{1}\right)-w(i)}=1 / m$. We show that $m$ is no bigger than 6 . Consider the triangle $\triangle i i_{1} i_{2}$. We have $l_{12} / l_{1}=e^{w\left(i_{1}\right)-w(i)}=$ $1 / m$. By the triangle inequality, $l_{2}>(m-1) l_{12}$, and thus $e^{w\left(i_{2}\right)-w(i)}=l_{12} / l_{2}<$ $1 /(m-1)$. Now consider the triangle $\triangle i i_{2} i_{3}$. Similarly we obtain $e^{w\left(i_{3}\right)-w(i)}<$ $1 /(m-2)$. We can continue to consider the triangles around $i$ and obtain

$$
e^{w\left(i_{4}\right)-w(i)}<1 /(m-3), \ldots, \quad \text { and } \quad e^{w\left(i_{6}\right)-w(i)}<1 /(m-5) .
$$

By contradiction, assume $m>6$. For any $1 \leq j \leq 6$, we have $e^{w\left(i_{j}\right)-w(i)}<1$ and $w(i)>w\left(i_{j}\right)$. Thus $l_{j, j+1}$ is the shortest side in $\triangle i i_{j} i_{j+1}$ and its corresponding angle $\theta_{i}^{j}$ is the smallest and thus less than $\pi / 3$. Then $K_{i}=2 \pi-\sum_{j=1}^{6} \theta_{i}^{j}>0$. This contradicts $K \equiv 0$. Therefore $m \leq 6$ and $e^{w\left(i_{1}\right)-w(i)}=1 / m>1 / 6$, and $w\left(i_{1}\right)-w(i) \geq \log (1 / 6)$.

\section{Properties of Quasi-harmonic Functions (Lemmas 2.2 and 2.3)}

4.1. Proof of Lemma 2.2. Consider any $j \in B(i, R-1)$, and let $j_{1}, j_{2}, \cdots, j_{6}$ be its six neighbors. Since $f$ is quasi-harmonic, there exist $m_{1}, m_{2}, \cdots, m_{6} \geq m$, satisfying $\sum_{k=1}^{6} m_{k}=1$ and $f(j)=\sum_{k=1}^{6} m_{k} f\left(j_{k}\right)$. Thus for any $k$, we have $M-f\left(j_{k}\right) \leq(M-f(j)) / m$. In other words, for any two neighboring vertex $j \backsim l$ with $j \in B(i, R-1)$, we have $M-f(l) \leq(M-f(j)) / m$. By induction, we can show that $M-f(j) \leq(M-f(i)) / m^{n}$ if $d(i, j)=n \in \mathbb{N}^{+}$. In particular, since $M-f(i)<\epsilon m^{R}$, for any $j \in B(i, R), M-f(j)<\epsilon$, i.e., $M-\epsilon \leq\left. f\right|_{B(i, R)}$.

4.2. Proof of Lemma 2.3. Let $m$ be a harmonic factor of both $f_{1}$ and $f_{2}$. Choose a proper $M_{2}$ such that $\left|f_{2}(i)\right|<M_{2}$ for any vertex $i$. Let $n$ be an integer larger than $2 M_{2} /\left(\epsilon m^{R}\right)$ and $R_{2}=n R$. Since $M$ is the least upper bound of $f_{1}$, there exists a vertex $i$ such that $f_{1}(i)>M-\epsilon m^{R_{2}}$. By Lemma 2.2, $\left.f_{1}\right|_{B\left(i, R_{2}\right)}>M-\epsilon$.

Let $F(k)$ be the maximum of $f_{2}$ in $B(i, k R)$, i.e. $F(k)=\max _{j \in B(i, k R)} f_{2}(j)(k=$ $0,1, \ldots, n)$. By the definition of $F$, we have $-M_{2} \leq F(0) \leq F(1) \leq \cdots \leq F(n) \leq$ $M_{2}$. So there exists $k \in\{1,2, \ldots, n\}$ such that $F(k)-F(k-1) \leq 2 M_{2} / n \leq \epsilon m^{R}$. Choose $j_{0} \in B(i,(k-1) R)$ s.t. $f_{2}\left(j_{0}\right)=F(k-1) \geq F(k)-\epsilon m^{R}$. For $B\left(j_{0}, R\right) \subseteq$ $B(i, k R)$, we have $\left.f_{2}\right|_{B\left(j_{0}, R\right)} \leq F(k)$. By Lemma 2.2. $\left.f_{2}\right|_{B\left(j_{0}, R\right)} \geq F(k)-\epsilon$. Let $N=F(k)$ and we have $N-\epsilon \leq\left. f_{2}\right|_{B\left(j_{0}, R\right)} \leq N$. As $B\left(j_{0}, R\right) \subseteq B\left(i, R_{2}\right)$, we also have $M-\epsilon \leq\left. f_{1}\right|_{B\left(j_{0}, R\right)}<M$. We can just take $j_{0}$ here as $i$ in the lemma. 

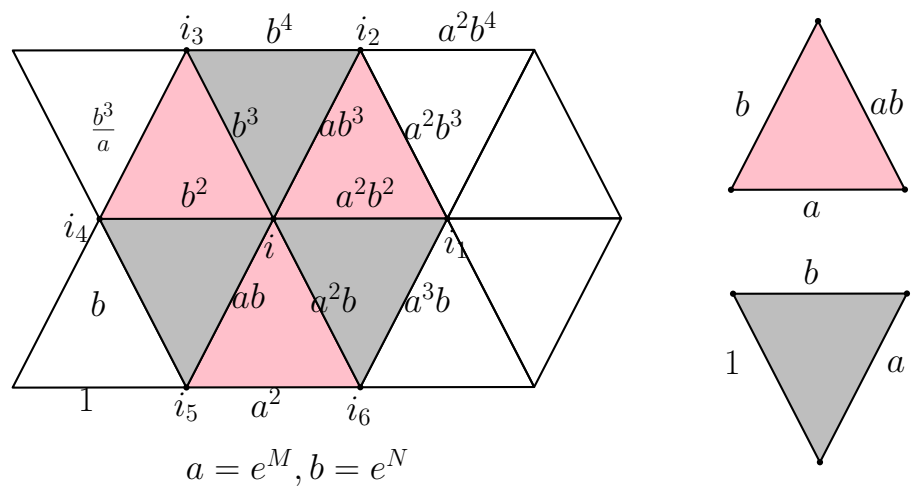

FiguRE 7 . In the case where $w$ is linear, there are only two types of triangles in the hexagonal mesh up to a similar transformation. (Color available online.)

\section{Consequences of $w$ Being (almost) linear}

(LEMmas 2.5, 2.6 AND 2.7)

5.1. Proof of Lemma 2.5. Since $w$ is linear, there exist $M$ and $N$ such that $\Delta_{1} w \equiv M$ and $\Delta_{\omega} w \equiv N$. One can show that for any vertex $i, \triangle i i_{1} i_{2}, \triangle i_{4} i i_{3}$ and $\triangle i_{5} i_{6} i$ are similar to each other, and thus $\theta_{i}^{1}+\theta_{i}^{3}+\theta_{i}^{5}=\pi$ (see Figure 7). For the same reason, $\theta_{i}^{2}+\theta_{i}^{4}+\theta_{i}^{6}=\pi$. Therefore $K_{i}=0$ for any vertex $i \in V$.

\subsection{Proof of Lemma 2.6.}

Lemma 5.1. Assume we have an immersion g from $\Sigma$ to $\mathbb{C}$, and we take a vertex in $V$ just as the points in $\Sigma$, with unit vector $e \in\{ \pm 1, \pm \omega, \pm(\omega+1)\}$. For any $i \in V$, we have a unique orientation preserving similar transformation (non-degenerated 1-dimension complex affine transformation) $T$ on $\mathbb{C}$ such that for any $k \in \mathbb{Z}$, $T(g(i+k e))=g(i+(k+1) e)$.

Proof. From the proof of Lemma 2.5, there are two types of triangles up to similar transformation in the triangulation, which means the angle between vector

$$
\overrightarrow{g(i+(t-1) e) g(i+t e)}, \overrightarrow{g(i+t e) g(i+(t+1) e)}
$$

is independent of $t$. Here $t$ is an integer. By the linearity of $w$, one can also verify that

$$
|\overrightarrow{g(i+(t-1) e) g(i+t e)}| /|\overrightarrow{g(i+t e) g(i+(t+1) e)}|
$$

is independent of $t$. Thus there exists the unique $k \in \mathbb{C}$ such that

$$
(g(i+(t+1) e)-g(i+t e))=k(g(i+t e)-g(i+(t-1) e)) .
$$

If we denote $b=g(i+(t+1) e)-k g(i+t e)=g(i+t e)-k g(i+(t-1) e)$, then $b$ is a constant independent of $t$. Therefore $g(i+(t+1) e)=k g(i+t e)+b$, and the transformation $T: z \mapsto k z+b$ is the unique map as we claimed.

Assume we have an immersion $g$ from $\Sigma$ to $\mathbb{C}$. From the hypothesis, $w$ is linear on $V_{0}$. For any $i \in V$, there are at least two unit vectors $e_{1}, e_{2} \in\{ \pm 1, \pm \omega, \pm(\omega+1)\}$ 
such that $w\left(i+e_{1}\right)-w(i)<0, w\left(i+e_{2}\right)-w(i)<0$. By Lemma 5.1, there exists the unique contract affine transformation $T_{1}$ on $\mathbb{C}$ which maps $g\left(i+t e_{1}\right)$ to $g\left(i+(t+1) e_{1}\right)$ and has the unique fixed point denoted $O_{1}$. Similarly, there exists the unique contract affine transformation $T_{2}$ on $\mathbb{C}$ which maps $g\left(i+t e_{2}\right)$ to $g\left(i+(t+1) e_{2}\right)$ and has the unique fixed point denoted $\mathrm{O}_{2}$.

For $\left\|T_{1}\right\|=e^{2\left(w\left(i+e_{1}\right)-w(i)\right)}<1,\left\|T_{2}\right\|=e^{2\left(w\left(i+e_{2}\right)-w(i)\right)}<1$, by the fixed point theorem $T_{1}^{m}(g(i)) \rightarrow O_{1}, T_{2}^{m}(g(i)) \rightarrow O_{2}$ as $m \rightarrow \infty$.

$$
\begin{aligned}
& \left|O_{1}-O_{2}\right| \\
= & \lim _{m \rightarrow \infty}\left|T_{1}^{m}(g(i))-T_{2}^{m}(g(i))\right| \\
= & \lim _{m \rightarrow \infty}\left|g\left(i+m e_{1}\right)-g\left(i+m e_{2}\right)\right| \\
\leq & \lim _{m \rightarrow \infty}\left(\sum_{s=1}^{m}\left|g\left(i+m e_{1}+(s-1) e_{2}\right)-g\left(i+m e_{1}+s e_{2}\right)\right|\right. \\
& \left.+\sum_{s=1}^{m}\left|g\left(i+m e_{2}+(s-1) e_{1}\right)-g\left(i+m e_{2}+s e_{1}\right)\right|\right) \\
\leq & \lim _{m \rightarrow \infty}\left(m\left|g\left(i+m e_{1}\right)-g\left(i+m e_{1}+e_{2}\right)\right|+m\left|g\left(i+m e_{2}\right)-g\left(i+m e_{2}+e_{1}\right)\right|\right) \\
= & \lim _{m \rightarrow \infty} m\left(\left\|T_{1}\right\|_{2}^{m}\left|g(i)-g\left(i+e_{2}\right)\right|+|| T_{2} \|_{2}^{m}\left|g(i)+g\left(i+e_{1}\right)\right|\right) \\
= & \left.0 \quad \quad \quad \text { for }|| T_{1}\|,\| T_{2} \|<1\right) .
\end{aligned}
$$

So $O_{1}=O_{2}$. We may assume it is the origin and $T_{1}(z)=r_{1} e^{2 \pi i \alpha_{1}} z, T_{2}(z)=$ $r_{2} e^{2 \pi i \alpha_{2}} z$. There exist $m_{s} \rightarrow+\infty, n_{s} \rightarrow+\infty$ such that $r_{1}^{m_{s}} r_{2}^{-n_{s}} \rightarrow 1$. And for any $\epsilon>0$, because $\left\{\left|T_{2}^{-n_{s}} T_{1}^{m_{s}}(g(i))\right|: \forall s\right\}$ is bounded, there exists $s \neq t$ such that $\left|T_{2}^{-n_{s}} T_{1}^{m_{s}}(g(i))-T_{2}^{-n_{t}} T_{1}^{m_{t}}(g(i))\right|<\epsilon / 2$ and $r_{1}^{m_{s}} r_{2}^{-n_{s}}>1 / 2$, and thus $\left|g(i)-T_{2}^{n_{s}-n_{t}} T_{1}^{m_{t}-m_{s}}(g(i))\right|<\epsilon$. Choose $R=\left|n_{s}-n_{t}\right|+\left|m_{t}-m_{s}\right|$ and then $T_{2}^{n_{s}-n_{t}} T_{1}^{m_{t}-m_{s}} g(i) \in B(i, R)$, and when $\epsilon$ is small enough, $T_{2}^{n_{s}-n_{t}} T_{1}^{m_{t}-m_{s}} g(i)$ must be in the hexagonal neighborhood of $g(i)$ and this indicates overlapping.

5.3. Proof of Lemma 2.7. For the sake of convenience, assume $i=0$ and $w(0)=$ 0 . Otherwise one can perform a similar transformation to the mesh to make $w(0)=$ 0 . For any positive integer $R$, let

$$
\begin{aligned}
& W_{R}=\{w: B(0, R) \rightarrow \mathbb{R} \mid w(0)=0\} \\
& W_{R}^{f}=\left\{w \in W_{R} \mid w \text { induces zero curvature in } B(0, R)\right\}, \text { and } \\
& W_{R}^{o}=\left\{w \in W_{R}^{f} \mid \text { The immersion of } B(0, R)\right. \text { into the plane has an } \\
& \text { overlap of positive area }\} .
\end{aligned}
$$

$W_{R}$ is a linear space of finite dimension and can be equipped with a metric, for instance, induced from $L_{2}$ norm. $W_{R}^{f}$ and $W_{R}^{o}$ are two subsets (not necessarily subspaces) of $W_{R}$ and have a natural inherent metric. Based on Lemma 2.4, $W_{R}^{f}$ is bounded. When we immerse the mesh in $B(0, R)$ with $w \in W_{R}^{f}$ into the plane, we have the freedom of choosing a base point and the orientation of an edge incident to the base point. On the other hand, once they are chosen, the immersion is uniquely 
determined. By induction, one can show that the positions of the immersed vertices are continuous functions of $w$. In addition, whether the immersion has an overlap of position area is independent of the choice of the base point and the orientation of that edge. Therefore for any $w \in W_{R}^{o}$, any sufficiently small perturbation of $w$ in $W_{R}^{f}$ will not move $w$ out of $W_{R}^{o}$. So $W_{R}^{o}$ is an open subset of $W_{R}^{f}$.

According to the hypothesis of the lemma, we fix $M>0$. For any $N$, let $w_{M, N}$ be the linear PL conformal factor i.e., $\Delta_{1} w_{M, N} \equiv M$ and $\Delta_{\omega} w_{M, N} \equiv N$. By Lemma 2.5] $\left.w_{M, N}\right|_{B(0, R)} \in W_{R}^{f}$ for any $R$. For simplicity, when it is clear from the context, we also denote $w_{M, N}$ as its restriction to $B(0, R)$. By Lemma 2.6, we know for any $N$ there exists $R(N)$ large enough such that $w_{M, N} \in W_{R(N)}^{o}$. Since $W_{R(N)}^{o}$ is open in $W_{R(N)}^{f}$, there exists a neighborhood of $w_{M, N}$ in $W_{R}^{f}(N)$ which remains in $W_{R(N)}^{o}$. In particular, there exists $\epsilon(N)>0$ sufficiently small such that for any $N^{\prime} \in(N-\epsilon(N), N+\epsilon(N))$, any $w \in W_{R(N)}^{f}$ satisfying $\left|\Delta_{1} w-M\right|<\epsilon(N)$ and $\left|\Delta_{\omega} w-N^{\prime}\right|<\epsilon(N)$ is still in $W_{R(N)}^{o}$.

So far both $\epsilon(N)$ and $R(N)$ depend on $N$. To obtain $\epsilon$ and $R$ independent on $N$ as claimed in the lemma, our strategy is to show that all possible $N$ form a compact set. Then based on the above results, we have an open covering of this compact set using the intervals $(N-\epsilon(N), N+\epsilon(N))$ for any $N$. From the compactness, we have a finite subcover and thus obtain a uniform $\epsilon$ and $R$ independent on $N$.

Let $\widetilde{S}$ denote an open set such that $S \subseteq \widetilde{S} \subseteq \overline{\widetilde{S}} \subseteq(0, \pi)$, and define a set

\section{$N_{M}=\{N \mid$ all inner angles in the hexagonal mesh}

with the conformal factor $w_{M, N}$ are in $\left.\overline{\tilde{S}}\right\}$.

We claim that there exists $\epsilon_{0} \geq 0$ such that for any $\epsilon \leq \epsilon_{0}$ and any $R>2$, if $N$ is a real number so that there exists a conformal factor $w$ satisfying the hypotheses of the lemma, in particular including that

(i) the metric induced by $w$ is flat,

(ii) all inner angles are in $S$,

(iii) $M-\epsilon \leq\left.\Delta_{1} w\right|_{B(i, R)} \leq M$, and

(iv) $N-\epsilon \leq\left.\Delta_{\omega} w\right|_{B(i, R)} \leq N$,

then $N \in N_{M}$. This indeed shows $N_{M}$ is the set of all possible $N$.

To prove the above claim, consider the following two triangles $\triangle i(i-1)(i+\omega)$ and $\triangle i(i+1+\omega)(i+\omega)$ (see Figure 8). The inner angles of $\triangle i(i-1)(i+\omega)$ are continuous functions of $\Delta_{1} w(i-1)$ and $\Delta_{\omega} w(i)$, and the inner angles of $\triangle i(i+1+\omega)(i+\omega)$ are continuous functions of $\Delta_{1} w(i+\omega)$ and $\Delta_{\omega} w(i)$. Denote

$D_{1}=\left\{\left(\Delta_{1} w(i-1), \Delta_{\omega} w(i)\right) \mid\right.$ the inner angles of $\triangle i(i-1)(i+\omega)$ are in $\left.S\right\}$,

$D_{2}=\left\{\left(\Delta_{1} w(i+\omega), \Delta_{\omega} w(i)\right) \mid\right.$ the inner angles of $\triangle i(i+1+\omega)(i+\omega)$ are all in $\left.S\right\}$,

$\widetilde{D_{1}}=\left\{\left(\Delta_{1} w(i-1), \Delta_{\omega} w(i)\right) \mid\right.$ the inner angles of $\triangle i(i-1)(i+\omega)$ are all in $\left.\tilde{S}\right\}$, and

$\widetilde{D_{2}}=\left\{\left(\Delta_{1} w(i+\omega), \Delta_{\omega} w(i)\right) \mid\right.$ the inner angles of $\triangle i(i+1+\omega)(i+\omega)$ are all in $\left.\tilde{S}\right\}$. 


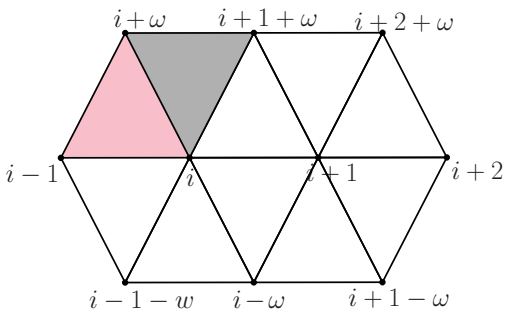

Figure 8. Illustration for the proof of Lemma 2.7. (Color available online.)

By Lemma 2.4 we know that $D_{1}, D_{2}$ are bounded in $\mathbb{R}^{2}$. As $S$ is compact and $\tilde{S}$ is open, $D_{1}$ and $D_{2}$ are closed and thus compact, and $\widetilde{D_{1}}, \widetilde{D_{2}}$ are open neighborhoods of $D_{1}$ and $D_{2}$, respectively. So $d\left(D_{1}, \widetilde{D}_{1}^{c}\right)>0, d\left(D_{2}, \widetilde{D}_{2}^{c}\right)>0$. Choose $\epsilon_{0}<$ $\frac{1}{2} \min \left\{d\left(D_{1},{\widetilde{D_{1}}}^{c}\right), d\left(D_{2},{\widetilde{D_{2}}}^{c}\right)\right\}$.

Now for any $R \geq 2$ and any $\epsilon<\epsilon_{0}$, let $N$ be a number so that there exists a conformal factor $w$ satisfying the above hypotheses (i, ii, iii, iv). Then we have $\left(\Delta_{1} w(i-1), \Delta_{\omega} w(i)\right) \in D_{1}$ and $d\left(\left(\Delta_{1} w(i-1), \Delta_{\omega} w(i)\right),(M, N)\right)<2 \epsilon \leq 2 \epsilon_{0}<$ $d\left(D_{1},{\widetilde{D_{1}}}^{c}\right)$, Thus $(M, N) \in \widetilde{D_{1}}$. Similarly, $(M, N) \in \widetilde{D_{2}}$. Note that from the proof of Lemma 2.5, in a hexagonally triangulated plane conformal to a regular one with a linear conformal factor $w_{N, M}$, there are only two types of triangles up to similar transformation, which can be represented by $\triangle i(i-1)(i+\omega)$ and $\triangle i(i+1+\omega)(i+\omega)$. Thus we have $N \in N_{M}$.

According to the discussion above, for any $N \in N_{M}$ there exist $R(N)$ and $\epsilon(N)$ such that for any $N^{\prime} \in(N-\epsilon(N), N+\epsilon(N))$, if $w \in W_{R(N)}^{f}$ satisfies $\left|\Delta_{1} w-M\right|<$ $\epsilon(N)$ and $\left|\Delta_{\omega} w-N^{\prime}\right|<\epsilon(N)$, then $w$ is in $W_{R(N)}^{o}$. Now $\bigcup_{N \in N_{M}}(N-\epsilon(N), N+\epsilon(N))$ is an open cover of $N_{M}$ and there exists a finite subcover $\bigcup_{j=1}^{n} B\left(N_{j}, \epsilon\left(N_{j}\right)\right)$. Choose

$$
\epsilon=\min \left\{\epsilon_{0}, \epsilon\left(N_{1}\right), \cdots, \epsilon\left(N_{n}\right)\right\} \text { and } R=\max \left\{2, R\left(N_{1}\right), \cdots, R\left(R_{n}\right)\right\} .
$$

Finally, for any $N$, if $w$ satisfies the above hypotheses (i, ii, iii, iv) for the chosen $\epsilon$ and $R$, from the above claim, we have that $N \in N_{M}$. Thus $N \in\left(N_{j}-\epsilon\left(N_{j}\right)\right.$, $\left.N_{j}-\epsilon\left(N_{j}\right)\right)$ for some $1 \leq j \leq n$. By the choice of $\epsilon$ and $R$, we have $w \in W_{R\left(N_{j}\right)}^{f}$ and $\left|\Delta_{1} w-M\right|<\epsilon\left(N_{j}\right)$ and $\left|\Delta_{\omega} w-N\right|<\epsilon\left(N_{j}\right)$. This implies $w \in W_{R}^{o}$ and proves the lemma.

\section{REFERENCES}

[1] A. Bobenko, U. Pinkall, and B. Springborn, Discrete conformal maps and ideal hyperbolic polyhedra, arXiv:1005.2698 [math.GT], May 2010.

[2] Zheng-Xu He, An estimate for hexagonal circle packings, J. Differential Geom. 33 (1991), no. 2, 395-412. MR1094463 (92b:52039)

[3] Zheng-Xu He, Rigidity of infinite disk patterns, Ann. of Math. (2) 149 (1999), no. 1, 1-33, DOI 10.2307/121018. MR.1680531 (2000j:30068)

[4] Feng Luo, Private communication.

[5] Feng Luo, Combinatorial Yamabe flow on surfaces, Commun. Contemp. Math. 6 (2004), no. 5, 765-780, DOI 10.1142/S0219199704001501. MR2100762 (2005m:53122)

[6] Burt Rodin and Dennis Sullivan, The convergence of circle packings to the Riemann mapping, J. Differential Geom. 26 (1987), no. 2, 349-360. MR906396 (90c:30007) 
[7] Oded Schramm, Rigidity of infinite (circle) packings, J. Amer. Math. Soc. 4 (1991), no. 1, 127-149, DOI 10.2307/2939257. MR1076089(91k:52027)

Mathematical Sciences Center, Tsinghua University, Beijing 100084, People's RepubLIC OF CHINA

E-mail address: mike890505@gmail.com

Department of Computer Science, Stony Brook University, Stony Brook, New York 11794

E-mail address: gu@cs.stonybrook.edu

Mathematical Sciences Center, Tsinghua University, Beijing 100084, People's RepubLIC OF CHINA

E-mail address: jsun@math.tsinghua.edu.cn 